\title{
Experimental evidence of a change of exchange anisotropy sign with temperature in $\mathrm{Zn}$-substituted $\mathrm{Cu}_{2} \mathrm{OSeO}_{3}$
}

\author{
S. H. Moody,,${ }^{1,}$ P. Nielsen, ${ }^{1}$ M. N. Wilson, ${ }^{1}$ D. Alba Venero $\odot,{ }^{2}$ A. Štefančič ${ }^{3}$ G. Balakrishnan $\odot,{ }^{3}$ and P. D. Hatton ${ }^{1}$ \\ ${ }^{1}$ Durham University, Department of Physics, South Road, Durham DH1 3LE, United Kingdom \\ ${ }^{2}$ ISIS Neutron and Muon Source, Rutherford Appleton Laboratory, Didcot OX11 OQX, United Kingdom \\ ${ }^{3}$ University of Warwick, Department of Physics, Coventry CV4 7AL, United Kingdom
}

(Received 20 May 2021; revised 27 October 2021; accepted 27 October 2021; published 1 December 2021)

\begin{abstract}
We report small-angle neutron scattering from the conical state in a single crystal of $\mathrm{Zn}$-substituted $\mathrm{Cu}_{2} \mathrm{OSeO}_{3}$. Using a $3 \mathrm{D}$ vector-field magnet to reorient the conical wave vector, our measurements show that the magnitude of the conical wave vector changes as a function of crystallographic direction. These changes are explained using the anisotropic exchange interaction (AEI) within the continuum model, whose magnitude in free-energy transitions from a maxima to a minima along the $\langle 111\rangle$ and $\langle 100\rangle$ crystallographic directions respectively. We further find that the AEI free-energy constant undergoes a change of sign from positive to negative with decreasing temperature. Unlike in the related compound FeGe, where similar behavior of the AEI induces a reorientation of the helical wave vector, we show that the zero field helical wave vector in $\left(\mathrm{Cu}_{0.98} \mathrm{Zn}_{0.02}\right)_{2} \mathrm{OSeO}_{3}$ remains along the $\langle 100\rangle$ directions at all temperatures due to the competing fourth-order magnetocrystalline anisotropy becoming dominant at lower temperatures.
\end{abstract}

DOI: 10.1103/PhysRevResearch.3.043149

The lack of inversion symmetry in the family of B20 chiral cubic materials allows a nonvanishing DzyaloshinskiiMoriya interaction (DMI), which competes with numerous other magnetic interactions to stabilize a wide variety of incommensurate magnetic states [1-8]. At zero field and at temperatures below $T_{\mathrm{C}}$, the isotropic exchange interaction and DMI (which prefer spin-aligned with constant $A$ and spin perpendicular with constant $D$, respectively) twist the magnetic texture into a helix with a periodicity determined by the ratio of the strengths of the two interactions, $q \approx D / A$. Within a mean-field continuum model, the cubic crystal structure allows higher-order anisotropic couplings beyond the DMI to be described by a single constant $\gamma$, commonly named the anisotropic exchange interaction (AEI) [9]. In MnSi, the helical wave vector was shown to align along the $\langle 111\rangle$ directions [10] due to a negative AEI constant [11-13]. $\gamma$ is also known to be temperature dependent, with a change of sign with temperature being responsible for the helical wave vector reorientation in the related material, FeGe [14-16].

Since the AEI originates from higher-order interactions and is typically weak, the orientation of these incommensurate textures can easily be manipulated by the application of a magnetic field, which induces a transition from the multidomain magnetic helix into a single-domain magnetic conical state, whose wave vector aligns with the magnetic field [10].

\footnotetext{
*samuel.h.moody@durham.ac.uk

Published by the American Physical Society under the terms of the Creative Commons Attribution 4.0 International license. Further distribution of this work must maintain attribution to the author(s) and the published article's title, journal citation, and DOI.
}

Within the cone state, the spins cant towards the field direction to minimize the Zeeman interaction. Applying a higher field reduces the conical angle $\theta$, but maintains a constant wave vector $q$ until the cone angle reaches zero to form a forced ferromagnetic state [17].

The behavior of chiral magnets adopting a B20 structure materials near $T_{\mathrm{C}}$ is further enriched by thermal fluctuations, which induce a hexagonal lattice of magnetic skyrmions within small region at nonzero field. Magnetic skyrmions are vortex-like whirls of magnetization that are the $2 \mathrm{D}$ solitonic solutions to systems hosting a number of competing magnetic interactions [1,18-22]. Their particle-like nature and topological properties make magnetic skyrmions appealing for applications within a variety of spintronic devices, ranging from race-track memory schemes and logic devices, to stochastic and neuromorphic computing [23-29].

The effects of anisotropic interactions such as magnetocrystalline anisotropy (MCA) and the AEI on these magnetic textures are typically overlooked, as the inherent cubic symmetry of the B20 materials limits the strength of these higher-order energy terms [30]. Despite this, anisotropystabilised magnetic textures within the multiferroic material $\mathrm{Cu}_{2} \mathrm{OSeO}_{3}$ have been found to exist at low temperatures, such as the low-temperature skyrmion (LTS) [31-34] and tilted conical (TC) phases, where the conical wave vector deviates from the direction of the magnetic field applied along a [1 00 ], with $q$ canting towards the $\langle 111\rangle$ [35]. Using a continuum model, a negative AEI was found to be essential to prevent the TC texture from collapsing into a field-polarized state. Currently, the effects of the AEI have not been investigated on the LTS, and decoupling the effects of MCA and the AEI remains a challenge as the conventional method of ferromagnetic resonance uses high fields to investigate the field-polarized 


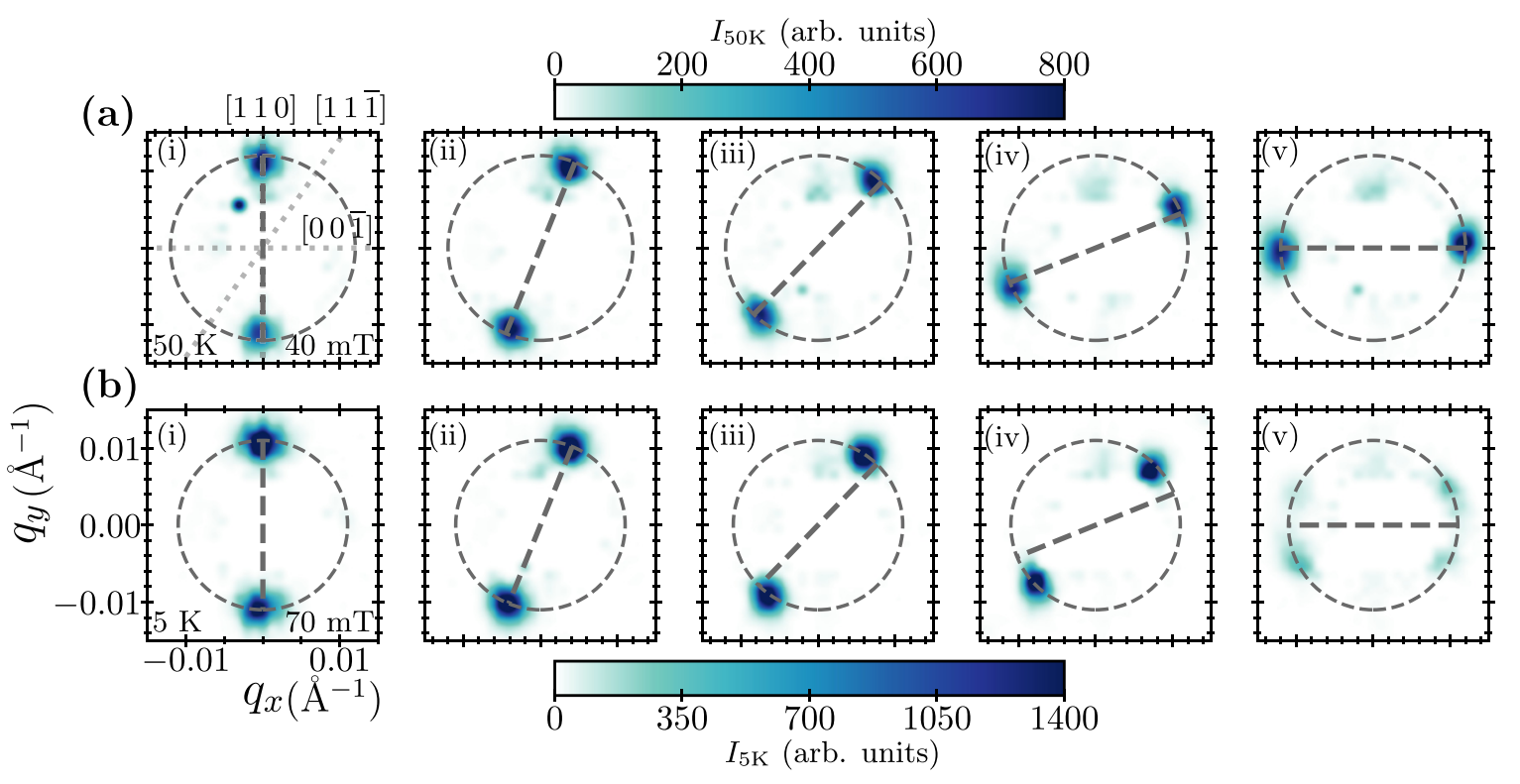

FIG. 1. Selection of reduced SANS patterns for field rotations at $40 \mathrm{mT}, 50 \mathrm{~K}$ (a) and $70 \mathrm{mT}, 5 \mathrm{~K}$ (b). The field angles for each frame are $0,22,44,68$, and $90^{\circ}$ for frames (i)-(v) and are shown by the thick dashed lines. The thin-dashed circle gives the location of equidistant $q=0.011 \AA^{-1}$. Crystallographic directions are shown by the dotted lines in (a)(i).

state where the effects of the AEI vanish [36]. Distinguishing and quantifying these anisotropic interactions is therefore important to better understand the low-temperature behavior of $\mathrm{Cu}_{2} \mathrm{OSeO}_{3}$.

In this paper, we utilize a $3 \mathrm{D}$ vector-field magnet together with small-angle neutron scattering (SANS) to show that the AEI free-energy constant changes sign from positive to negative with decreasing temperature in $\left(\mathrm{Cu}_{0.98} \mathrm{Zn}_{0.02}\right)_{2} \mathrm{OSeO}_{3}$. Unlike in the related compound $\mathrm{FeGe}$, this change of sign does not induce a reorientation of the zero-field magnetic helix. This demonstrates that the 4th order MCA also increases in magnitude with decreasing temperature, greater than the AEI to stabilize the zero-field magnetic helices along the $\langle 100\rangle$ directions at all temperatures [37]. Our findings provide insight into the low-temperature behavior of $\mathrm{Cu}_{2} \mathrm{OSeO}_{3}$ that will be essential for complete understanding of the creation and stabilization mechanisms of the recently discovered LTS and TC phases within pristine $\mathrm{Cu}_{2} \mathrm{OSeO}_{3}$.

A 20.5-mg single crystal of $\left(\mathrm{Cu}_{0.98} \mathrm{Zn}_{0.02}\right)_{2} \mathrm{OSeO}_{3}$ was grown at the University of Warwick utilizing the chemical vapour transport technique; see [38] for details. For the SANS experiment, the LARMOR instrument at the ISIS Pulsed Neutron and Muon Source was used. The sample was aligned with an x-ray Laue camera (Multiwire Laboratories) such that the $\left[\begin{array}{lll}1 & 1 & 0\end{array}\right]$ direction was vertical in the laboratory frame and the $\left[\begin{array}{lll}1 & -1 & 0\end{array}\right]$ direction was parallel with the incident neutron beam. This orientation allows the magnetic textures whose periodic components lie within a plane spanned by the all 3 high cubic-symmetry directions $(\langle 100\rangle,\langle 110\rangle$, and $\langle 111\rangle)$ to simultaneously satisfy the Bragg condition [39]. A zinc-substituted sample was chosen for our study due to the reduction of the exchange interaction compared to pristine $\mathrm{Cu}_{2} \mathrm{OSeO}_{3}$, resulting in a greater value of $q$ [37]. Previously, we have shown strong similarities of the magnetic behavior between $\mathrm{Zn}$-substituted and pristine samples [37], and hence we expect our results here will extend to pristine samples.
The time-of-flight (TOF) neutron diffraction data was reduced with Mantid [40], using neutron wavelengths between 0.9 and 13.0 ^, which were calibrated using a standard sample.

To observe the effects of the anisotropic interactions on the magnetic textures within $\mathrm{Cu}_{2} \mathrm{OSeO}_{3}$, we performed field scans by rotating the direction of the magnetic field at a constant magnitude. Initially, the field was applied vertically in the laboratory frame after zero-field cooling. The field orientation was then rotated 90 degrees in 46 steps about an axis parallel with the neutron beam, such that the $\left[\begin{array}{lll}1 & -1 & 0\end{array}\right]$, $\left[\begin{array}{lll}1 & 1 & -1\end{array}\right],\left[\begin{array}{lll}0 & 0 & -1\end{array}\right]$ directions were parallel with the field at angles of $0,35.3$, and 90 degrees, respectively. This procedure was performed at 5,12 , and $50 \mathrm{~K}$ at magnetic field magnitudes of 70,60 , and $40 \mathrm{mT}$, respectively. These fields were chosen to avoid phase coexistence between the magnetic conical and helical states; see Supplemental Material [41]. A selection of frames from the field scans are shown in Fig. 1(a).

In the first frame of the $50 \mathrm{~K}$ dataset, Fig. 1(a)(i), the vertically applied magnetic field induces a magnetic conical

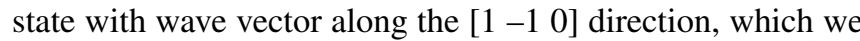
detect as a single pair of vertical peaks with $q \approx 0.011 \AA^{-1}$, corresponding to a real space length of $57 \mathrm{~nm}$. Upon rotating the field, Fig. 1(a)(ii-v), the conical wave vector rotates in an attempt to follow the direction of the magnetic field. This behavior is expected in the limit in which the AEI and MCA are much smaller than the isotropic Dzyaloshinskii-Moriya and exchange interactions $(\gamma, K \ll A, D)$, allowing the conical wave vector to rotate freely to minimize the Zeeman interaction. However, this behavior is not replicated within the data taken at lower temperatures in Fig. 1(b)(i-v), where angle of the conical wave vector $(\phi)$ lags behind the magnetic field angle $\left(H_{\phi}\right)$, particularly after the field passes through the [ $\left.\begin{array}{lll}1 & 1 & -1\end{array}\right]$ direction. This offset between the field direction and $q$ increases in magnitude with further rotation of the magnetic field, up to a maxima just before the field is applied along the $\left[\begin{array}{lll}0 & 0 & -1\end{array}\right]$ direction. This magnetic state is characteristic of 
(a)
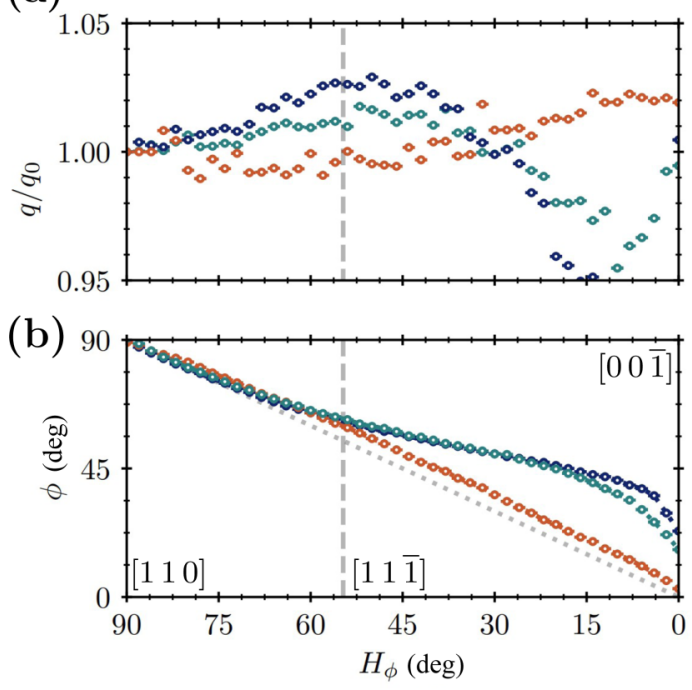

FIG. 2. (a) Normalised wave vector magnitude, and wave vector angle (b), $\phi$ in Fig. 1(a), of the conical state as a function of applied field angle $H_{\phi}$. Dark blue, light blue, and orange indicate temperatures of 5, 12, and $50 \mathrm{~K}$, respectively. Dashed line shows [ $\left.\begin{array}{lll}1 & 1 & -1\end{array}\right]$ direction. Dotted line shows $H_{\phi}$.

the tilted conical state seen previously in pristine $\mathrm{Cu}_{2} \mathrm{OSeO}_{3}$ [35], and shows that while isotropic interactions dominate at temperatures near $T_{\mathrm{C}}$, the anisotropic interactions become increasingly important at low temperatures and prevent free rotation of the magnetic conical state.

In order to quantify the effects of the anisotropic interactions, the conical peaks collected during the field scans shown in Fig. 2 and an additional set at $12 \mathrm{~K}$ were fit using two dimensional Gaussian functions in polar coordinates, allowing the extraction of the magnetic wave vector angle $\phi$ and magnitude $q$. The results of this fitting for field angle scans at the three different temperatures are shown in Fig. 2.

The behavior of $q$ as a function of field angle is shown in Fig. 2(a). A clear difference between the high temperature and low temperature regimes is observed. In the $50 \mathrm{~K}$ data set $q$ initially decreases slightly as the applied field is rotated from the [lllllll 110$]$ to $\left[\begin{array}{lll}1 & 1 & -1\end{array}\right]$ direction and then increases as the field is rotated further, up to a maximum when $\mathrm{H}$ is along $\left[\begin{array}{lll}0 & 0 & -1\end{array}\right]$. This behavior is reversed at low temperatures (both at $12 \mathrm{~K}$ and $5 \mathrm{~K}$ ), where the magnitude of the conical wave vector instead increases to a maximum when the field is rotated from

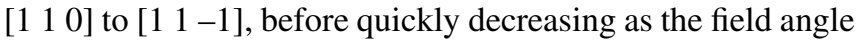
approaches the $\left[\begin{array}{lll}0 & 0 & -1\end{array}\right]$ direction. In the Fig. 2(b), the effects of the anisotropic interactions can also clearly be seen to be more significant in the low-temperature data sets, as in them $\phi$ deviates substantially from linearity after the magnetic field passes through [ $\left[\begin{array}{lll}1 & 1 & -1\end{array}\right]$, as compared to the $50 \mathrm{~K}$ data set where $\phi$ deviates only slightly from the expected linear trend in the absence of anisotropic interactions.

The dependence of $q$ on $H_{\phi}$ allows us to decouple the two dominant anisotropic interactions. We start by using the nonconstant terms within the free energy expansion derived by Bak and Jensen [11], which is valid for systems of $P 2_{1} 3$ crystal symmetry that host slowly-varying magnetization den- sities within the continuum approximation $\mathbf{m}(\mathbf{r})$ [42]:

$$
\begin{aligned}
F(\mathbf{r})= & D \mathbf{m}(\mathbf{r}) \cdot(\boldsymbol{\nabla} \times \mathbf{m}(\mathbf{r})) \\
& +\frac{1}{2} A\left[\left(\nabla m_{x}\right)^{2}+\left(\nabla m_{y}\right)^{2}+\left(\nabla m_{z}\right)^{2}\right] \\
& +\frac{1}{2} \gamma\left[\left(\frac{\partial m_{x}}{\partial x}\right)^{2}+\left(\frac{\partial m_{y}}{\partial y}\right)^{2}+\left(\frac{\partial m_{z}}{\partial z}\right)^{2}\right] \\
& +K\left(m_{x}^{4}+m_{y}^{4}+m_{z}^{4}\right)+\mu_{0}\left(\mathbf{m} \cdot \mathbf{H}_{\mathbf{a}}\right),
\end{aligned}
$$

where $D, A$ are the familiar Dzyaloshinskii-Moriya and exchange stiffness, and $K, \gamma$ are the 4th order MCA and AEI constants, respectively. The final term in Eq. (1) is the Zeeman interaction within an applied field $\mathbf{H}_{\mathbf{a}}$. In the case of $D=0$, and $A, \gamma>0$, the spin-texture reduces to a simple ferromagnet, with the spin direction given by the sign of the MCA constant $K$. We approximate a model spin-texture using a conical ansatz:

$$
\mathbf{m}(\mathbf{r}) / M_{s}=\sin \theta\left(\cos (\mathbf{q} \cdot \mathbf{r}) \hat{\mathbf{e}}_{1}+\sin (\mathbf{q} \cdot \mathbf{r}) \hat{\mathbf{e}}_{2}\right)+\cos \theta \hat{\mathbf{e}}_{3},
$$

where $\theta$ is the conical angle and $\left\{\hat{\mathbf{e}}_{n}\right\}$ define three mutually orthogonal basis vectors, with $\hat{\mathbf{e}}_{3} \| \mathbf{q}$. To compare with our experimental observations, we consider a conical texture with the wave vector restricted to the plane spanned by the three high cubic-symmetry directions, such that $\mathbf{q}=$ $\left(\frac{q}{\sqrt{2}} \sin \phi, \frac{q}{\sqrt{2}} \sin \phi, q \cos \phi\right)$. Using this form for the magnetic wave vector and substituting (2) into (1), integrating over one conical period $\lambda$ and differentiating with respect to $q$ we find a single stable solution:

$$
\begin{aligned}
\frac{\partial}{\partial q} \bar{F}(q, \phi) & =\sin ^{2} \theta\left[D+A q+\frac{1}{2} \gamma q \sin ^{2} \phi\left(3 \cos ^{2} \phi+1\right)\right] \\
q & =\frac{|D|}{A+\frac{1}{2} \gamma \sin ^{2} \phi\left(3 \cos ^{2} \phi+1\right)} .
\end{aligned}
$$

As shown by Eq. (3), the free energies which determine the periodicity of the incommensurate states within noncentrosymmetric magnets at a mean-field level are limited to the DMI and both the isotropic and anisotropic exchange interactions. Due to the the integration and normalisation of the free energy over an entire conical period, the Zeeman and anisotropy energies vanish due to the symmetry of the magnetic ansatz, and thus changing the magnitude of the applied field has no effect on the conical period. However, the direction of the applied field $\left(H_{\phi}\right)$ induces a rotation of the conical wave vector angle $(\phi)$ to follow the field direction, thus subjecting the conical state so the anisotropic effects of the AEI. The implications of this result can be seen in Figs. 3(a)-3(c), which show the experimentally measured values of the conical wave vector $q$ for different conical wave vector angles, $\phi$, at 50,12 , and $5 \mathrm{~K}$, respectively. At $50 \mathrm{~K}$, the increased wave vector at $\left[\begin{array}{lll}0 & 0 & -1\end{array}\right]$ compared to $\left[\begin{array}{lll}1 & 1 & -1\end{array}\right]$ is a clear indication of a positive exchange anisotropy constant $\gamma$, where energy costs for directions other than the $\langle 100\rangle$, are compensated by a shrinking of the conical wave vector. This behavior reverses with decreasing temperature, shown in Figs. 3(b) and 3(c), where the wave vector maxima are located at the [ $\left.\begin{array}{lll}1 & 1 & -1\end{array}\right]$ and minima towards the [0 $\left.0-1\right]$, suggesting a change of sign of $\gamma$. 


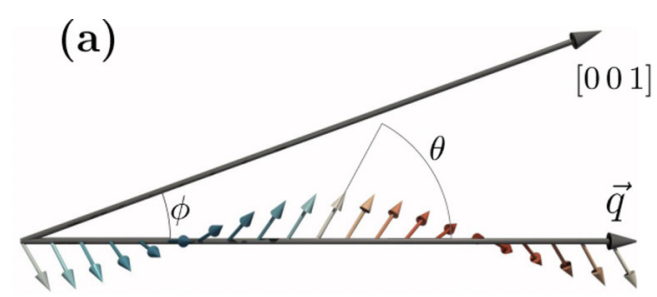

(b)

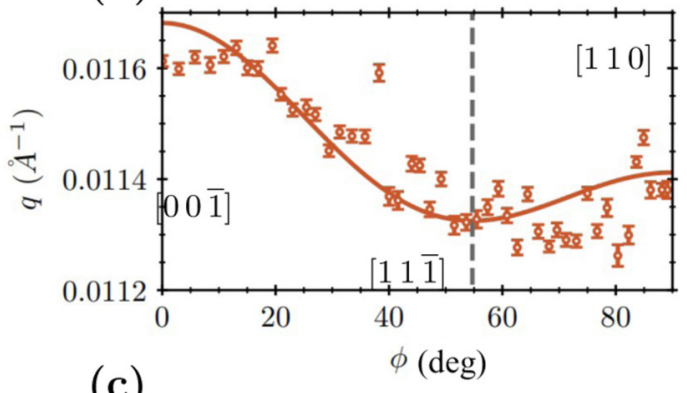

(c)

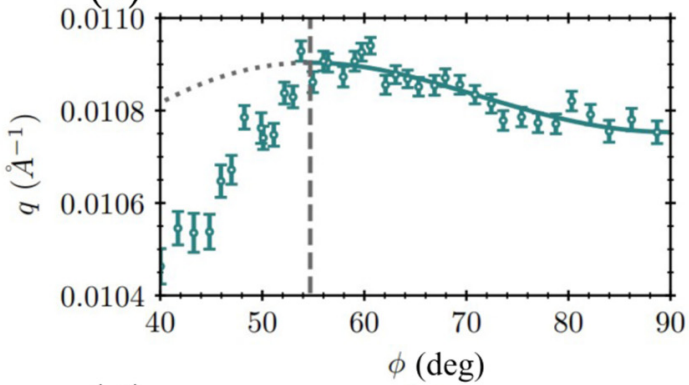

(d)
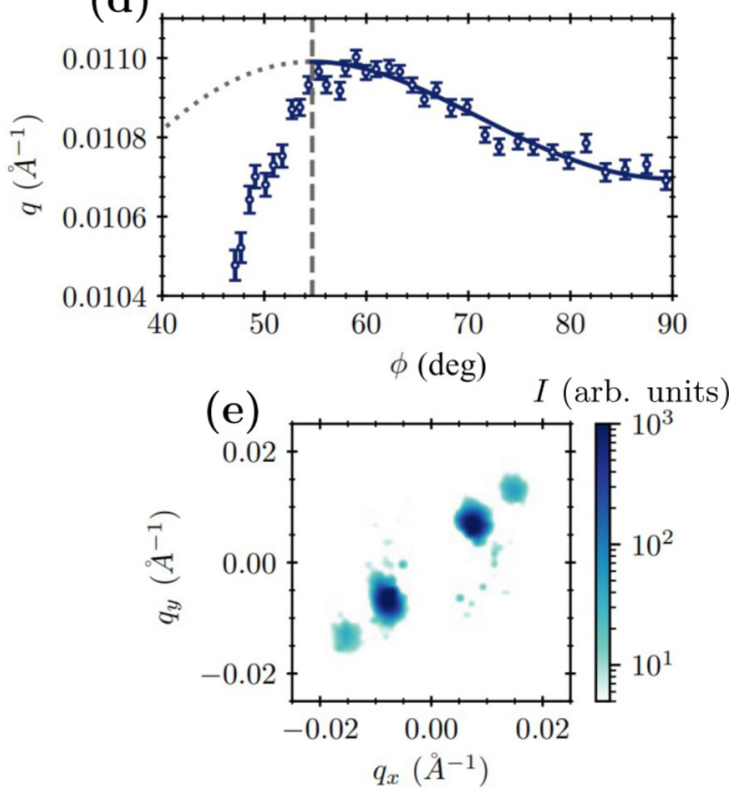

FIG. 3. (a) Schematic of a magnetic conical state with cone angle $\theta$ and wave vector $(\overrightarrow{\boldsymbol{q}})$ angle $\phi$. Values of the extracted wave vector magnitude $(q)$ against wave vector angle $(\phi)$ with $[(\mathrm{b})-(\mathrm{d})]$ being the 50,12 , and $5 \mathrm{~K}$ data sets, respectively. Fits to Eq. (3) are shown by the bold lines, with dotted lines showing regions ignored in the fitting procedure due to the appearance of higher-order peaks, shown in (e).

Using the experimentally determined value for the exchange stiffness, $A=4.4 \times 10^{-13} \mathrm{~J} / m$ from $T_{\mathrm{C}}=57 \mathrm{~K}$ $[38,43]$, and fitting both $D$ and $\gamma$ in (3) to the data in Fig. 3, we find $\gamma=2.1(2) \times 10^{-14} \mathrm{~J} / \mathrm{m}$ at $50 \mathrm{~K},-3.4(4) \times 10^{-14}$ at $12 \mathrm{~K}$, and $-6.7(3) \times 10^{-14} \mathrm{~J} / m$ at $5 \mathrm{~K}$. The low-temperature values are consistent with theoretical values of $\gamma$ required for TC formation [35], in agreement with the TC states observed in this paper.

During the refinement of the low-temperature data sets, only angles above 55 degrees were used. This is because at low temperatures, the direction of the conical wave vector deviates significantly from the magnetic field direction for $\phi<55$ degrees, inducing a component of the magnetic field perpendicular to the conical wave vector. Applying transverse fields to a helical state with a pinned $\mathbf{q}$ direction is known to deform the helix [44], introducing higher-order components to the Fourier transform of the magnetic state. The presence of higher order peaks in our SANS pattern shown in Fig. 3(e) suggest that similar deformations are occurring in our tilted conical state. For helices, these deformations are known to reduce the value of $q$ as the perpendicular field strength increases, which would be consistent with our observations of a large $\mathrm{q}$ discernibly where the higher order peaks are prominent. Fully accounting for these deformations within our model spin-texture remains an avenue intended for future study.

In the absence of cubic anisotropy, a change of sign of the AEI from positive to negative with decreasing temperature would induce a helical reorientation from the $\langle 100\rangle$ to $\langle 111\rangle$ directions. In order for the orientation of the helical wave vector to remain aligned along the $\langle 100\rangle$ directions (consistent with experimental observations), we find that $K$ must be negative, with the condition $|K|>\frac{4}{3}|\gamma| q^{2}$. This requires that the magnitude of $K$ increases with decreasing temperatures. This increase in $|K|$ at low temperatures is also in agreement with previous work discussing the origin of the low-temperature skyrmion state [33].

In conclusion, we performed SANS on a single crystal of $\left(\mathrm{Cu}_{0.98} \mathrm{Zn}_{0.02}\right)_{2} \mathrm{OSeO}_{3}$, using a novel $3 \mathrm{D}$ vector magnet to decouple the anisotropic interactions within the material by investigating the behavior of the magnetic conical state with a rotating magnetic field of constant magnitude. We observed a change of behavior of the magnitude of the conical wave vector $q$ as a function of wave vector angle $\phi$ whereby the crystal directions corresponding to a maximum or minimum in $q$ reversed with cooling from $50 \mathrm{~K}$ to $12 \mathrm{~K}$. We have explained this using a mean-field, continuum model and find that the AEI constant changes sign between these temperatures, with fitted values consistent with those required for tilted conical state formation. Unlike in the related compound FeGe, helical reorientation does not occur within $\mathrm{Cu}_{2} \mathrm{OSeO}_{3}$ with the change in sign of the AEI. This is due to an increasingly negative cubic anisotropy, which increases in magnitude faster than the AEI, causing the helical wave vectors to remain along the $\langle 100\rangle$ directions. We believe our finding that the AEI changes sign at similar temperatures to the occurrence of the LTS and TC magnetic phases will be highly useful for understanding the formation and stabilisation of these newly discovered magnetic textures within this material.

This work was supported by the UK Skyrmion Project EPSRC Programme Grant (No. EP/N032128/1). The SANS experiment at the ISIS Pulsed Neutron and Muon Source 
was supported by a beamtime allocation from the Science and Technology Facilities Council, Proposal No. 1920501.
M.N.W. acknowledges the support of the Natural Sciences and Engineering Research Council of Canada (NSERC).
[1] T. Lancaster, Skyrmions in magnetic materials, Contemp. Phys. 60, 246 (2019).

[2] I. E. Dzyaloshinskiŭ, Theory of helicoidal structures in antiferromagnets, J. Exptl. Theoret. Phys. (U.S.S.R.) 46, 1420 (1964).

[3] A. Neubauer, C. Pfleiderer, B. Binz, A. Rosch, R. Ritz, P. G. Niklowitz, and P. Böni, Topological Hall effect in the A Phase of MnSi, Phys. Rev. Lett. 102, 186602 (2009).

[4] T. Tanigaki, K. Shibata, N. Kanazawa, X. Yu, Y. Onose, H. S. Park, D. Shindo, and Y. Tokura, Real-space observation of short-period cubic lattice of skyrmions in mnge, Nano Lett. 15, 5438 (2015).

[5] H. Wilhelm, M. Baenitz, M. Schmidt, U. K. Rößler, A. A. Leonov, and A. N. Bogdanov, Precursor Phenomena at the Magnetic Ordering of the Cubic Helimagnet FeGe, Phys. Rev. Lett. 107, 127203 (2011).

[6] X. Z. Yu, N. Kanazawa, Y. Onose, K. Kimoto, W. Z. Zhang, S. Ishiwata, Y. Matsui, and Y. Tokura, Near room-temperature formation of a skyrmion crystal in thin-films of the helimagnet FeGe, Nat. Mater. 10, 106 (2011).

[7] S. Seki, X. Z. Yu, S. Ishiwata, and Y. Tokura, Observation of skyrmions in a multiferroic material, Science 336, 198 (2012).

[8] Y. Tokunaga, X. Z. Yu, J. S. White, H. M. Rønnow, D. Morikawa, Y. Taguchi, and Y. Tokura, A new class of chiral materials hosting magnetic skyrmions beyond room temperature, Nat. Commun. 6, 7638 (2015).

[9] O. Janson, I. Rousochatzakis, A. A. Tsirlin, M. Belesi, A. A. Leonov, U. K. Rößler, J. van den Brink, and H. Rosner, The quantum nature of skyrmions and half-skyrmions in $\mathrm{Cu}_{2} \mathrm{OSeO}_{3}$, Nat. Commun. 5, 5376 (2014).

[10] Y. Ishikawa, K. Tajima, D. Bloch, and M. Roth, Helical spin structure in manganese silicide $\mathrm{MnSi}$, Solid State Commun. 19, 525 (1976).

[11] P. Bak and M. H. Jensen, Theory of helical magnetic structures and phase transitions in MnSi and FeGe, J. Phys. C: Solid State Phys. 13, L881 (1980).

[12] O. Nakanishi, A. Yanase, A. Hasegawa, and M. Kataoka, The origin of the helical spin density wave in MnSi, Solid State Commun. 35, 995 (1980).

[13] S. V. Maleyev, Cubic magnets with Dzyaloshinskii-Moriya interaction at low temperature, Phys. Rev. B 73, 174402 (2006).

[14] B. Lebech, J. Bernhard, and T. Freltoft, Magnetic structures of cubic FeGe studied by small-angle neutron scattering, J. Phys.: Condens. Matter 1, 6105 (1989).

[15] M. L. Plumer, Wavevector and spin-flop transitions in cubic FeGe, J. Phys.: Condens. Matter 2, 7503 (1990).

[16] V. Ukleev, O. Utesov, L. Yu, C. Luo, K. Chen, F. Radu, Y. Yamasaki, N. Kanazawa, Y. Tokura, T.-H. Arima, and J. S. White, Signature of anisotropic exchange interaction revealed by vector-field control of the helical order in a FeGe thin plate, Phys. Rev. Research 3, 013094 (2021).

[17] Y. Ishikawa, T. Komatsubara, and D. Bloch, Magnetic phase diagram of MnSi, Phys. B: Condens. Matter 86-88, 401 (1977).

[18] K. Ishimoto, H. Yamauchi, Y. Yamaguchi, J. Suzuki, M. Arai, M. Furusaka, and Y. Endoh, Anomalous region in the magnetic phase diagram of (Fe,Co)Si, J. Magn. Magn. Mater. 90-91, 163 (1990).

[19] S. Mühlbauer, B. Binz, F. Jonietz, C. Pfleiderer, A. Rosch, A. Neubauer, R. Georgii, and P. Böni, Skyrmion lattice in a chiral magnet, Science 323, 915 (2009).

[20] U. K. Rößler, A. N. Bogdanov, and C. Pfleiderer, Spontaneous skyrmion ground states in magnetic metals, Nature (London) 442, 797 (2006).

[21] M. Vousden, M. Albert, M. Beg, M.-A. Bisotti, R. Carey, D. Chernyshenko, D. Corts-Ortuo, W. Wang, O. Hovorka, C. H. Marrows, and H. Fangohr, Skyrmions in thin films with easyplane magnetocrystalline anisotropy, Appl. Phys. Lett. 108, 132406 (2016).

[22] A. N. Bogdanov and C. Panagopoulos, Physical foundations and basic properties of magnetic skyrmions, Nat. Rev. Phys. 2, 492 (2020).

[23] X. Zhang, M. Ezawa, and Y. Zhou, Magnetic skyrmion logic gates: Conversion, duplication and merging of skyrmions, Sci. Rep. 5, 9400 (2015).

[24] A. Fert, N. Reyren, and V. Cros, Magnetic skyrmions: Advances in physics and potential applications, Nat. Rev. Mater. 2, 17031 (2017).

[25] S. L. Zhang, W. W. Wang, D. M. Burn, H. Peng, H. Berger, A. Bauer, C. Pfleiderer, G. van der Laan, and T. Hesjedal, Manipulation of skyrmion motion by magnetic field gradients, Nat. Commun. 9, 2115 (2018).

[26] A. F. Schäffer, L. Rózsa, J. Berakdar, E. Y. Vedmedenko, and R. Wiesendanger, Stochastic dynamics and pattern formation of geometrically confined skyrmions, Commun. Phys. 2, 72 (2019).

[27] H. Zhang, D. Zhu, W. Kang, Y. Zhang, and W. Zhao, Stochastic computing implemented by skyrmionic logic devices, Phys. Rev. Applied 13, 054049 (2020).

[28] S. Li, W. Kang, Y. Huang, X. Zhang, Y. Zhou, and W. Zhao, Magnetic skyrmion-based artificial neuron device, Nanotechnology 28, 31LT01 (2017).

[29] K. M. Song, J.-S. Jeong, B. Pan, X. Zhang, J. Xia, S. Cha, T.E. Park, K. Kim, S. Finizio, J. Raabe et al., Skyrmion-based artificial synapses for neuromorphic computing, Nat. Electron. 3, 148 (2020).

[30] W. Sucksmith and J. E. Thompson, The magnetic anisotropy of cobalt, Proc. R. Soc. London A 225, 362 (1954).

[31] A. Chacon, L. Heinen, M. Halder, A. Bauer, W. Simeth, S. Mühlbauer, H. Berger, M. Garst, A. Rosch, and C. Pfleiderer, Observation of two independent skyrmion phases in a chiral magnetic material, Nat. Phys. 14, 936 (2018).

[32] L. J. Bannenberg, R. Sadykov, R. M. Dalgliesh, C. Goodway, D. L. Schlagel, T. A. Lograsso, P. Falus, E. Lelièvre-Berna, A. O. Leonov, and C. Pappas, Skyrmions and spirals in MnSi under hydrostatic pressure, Phys. Rev. B 100, 054447 (2019).

[33] M. Halder, A. Chacon, A. Bauer, W. Simeth, S. Mühlbauer, H. Berger, L. Heinen, M. Garst, A. Rosch, and C. Pfleiderer, Thermodynamic evidence of a second skyrmion lattice phase 
and tilted conical phase in $\mathrm{Cu}_{2} \mathrm{OSeO}_{3}$, Phys. Rev. B 98, 144429 (2018).

[34] A. Aqeel, J. Sahliger, T. Taniguchi, S. Mändl, D. Mettus, H. Berger, A. Bauer, M. Garst, C. Pfleiderer, and C. H. Back, Microwave Spectroscopy of the Low-Temperature Skyrmion State in $\mathrm{Cu}_{2} \mathrm{OSeO}_{3}$, Phys. Rev. Lett. 126, 017202 (2021).

[35] F. Qian, L. J. Bannenberg, H. Wilhelm, G. Chaboussant, L. M. Debeer-Schmitt, M. P. Schmidt, A. Aqeel, T. T. M. Palstra, E. Brück, A. J. E. Lefering et al., New magnetic phase of the chiral skyrmion material $\mathrm{Cu}_{2} \mathrm{OSeO}_{3}$, Sci. Adv. 4, 9 (2018).

[36] M. Preißinger, K. Karube, D. Ehlers, B. Szigeti, H.-A. Krug von Nidda, J. S. White, V. Ukleev, H. M. Rønnow, Y. Tokunaga et al., Vital role of magnetocrystalline anisotropy in cubic chiral skyrmion hosts, npj Quantum Mater. 6, 65 (2021).

[37] M. T. Birch, S. H. Moody, M. N. Wilson, M. Crisanti, O. Bewley, A. Štefančič, G. Balakrishnan, R. Fan, P. Steadman, D. Alba Venero, R. Cubitt, and P. D. Hatton, Anisotropy-induced depinning in the $\mathrm{Zn}$-substituted skyrmion host $\mathrm{Cu}_{2} \mathrm{OSeO}_{3}$, Phys. Rev. B 102, 104424 (2020).

[38] A. Štefančič, S. H. Moody, T. J. Hicken, M. T. Birch, G. Balakrishnan, S. A. Barnett, M. Crisanti, J. S. O. Evans, S. J. R. Holt, K. J. A. Franke, P. D. Hatton, B. M. Huddart, M. R.
Lees, F. L. Pratt, C. C. Tang, M. N. Wilson, F. Xiao, and T. Lancaster, Origin of skyrmion lattice phase splitting in Zn-substituted $\mathrm{Cu}_{2} \mathrm{OSeO}_{3}$, Phys. Rev. Materials 2, 111402(R) (2018).

[39] S. Mühlbauer, D. Honecker, E. A. Périgo, F. Bergner, S. Disch, A. Heinemann, S. Erokhin, D. Berkov, C. Leighton, M. R. Eskildsen, and A. Michels, Magnetic small-angle neutron scattering, Rev. Mod. Phys. 91, 015004 (2019).

[40] O. Arnold, J. Bilheux, J. Borreguero, A. Buts, S. Campbell, L. Chapon, M. Doucet, N. Draper, R. Ferraz Leal, M. Gigg et al., Mantiddata analysis and visualization package for neutron scattering and $\mu$ SR experiments, Nucl. Instrum. Methods Phys. Res. Sect. A 764, 156 (2014).

[41] See Supplemental Material at http://link.aps.org/supplemental/ 10.1103/PhysRevResearch.3.043149 for gif images showing the full rotation-scan datasets.

[42] L. D. Landau and E. M. Lifshitz, Statistical Physics, 2nd ed. (Pergamon Press, Oxford, 1980).

[43] R. M. White, Quantum Theory of Magnetism (Springer, Berlin, 1983).

[44] Y. A. Izyumov, Modulated, or long-periodic, magnetic structures of crystals, Sov. Phys. Usp. 27, 845 (1984). 